Forum 2022 · 37:84-90

https://doi.org/10.1007/s12312-021-01031-x Online publiziert: 12. Januar 2022

(c) The Author(s), under exclusive licence to Springer Medizin Verlag GmbH, ein Teil von Springer Nature 2021

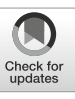

Beiträge der Autoren. Der Beitrag entstand durch das Autorenteam aus der Arbeitsgruppe Gesundheitspolitik und Market Access der Sektion C der Deutschen Krebsgesellschaft. Alle Autoren trugen in gleicher Weise zu Konzept, Entwicklung und Umsetzung des Manuskripts bei.

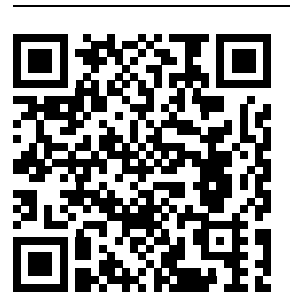

QR-Code scannen \& Beitrag online lesen

\section{Zehn Jahre AMNOG-Prozess aus Sicht der Onkologie}

\author{
Neuland erschlossen, Bebauung geht weiter
}

Arne Bartol · Katrin Dreßler · Peter Kaskel · Christiane Landsberg · Cornelia Lechner . Marco Petschulies

Arbeitsgruppe Gesundheitspolitik und Market Access der Sektion C der Deutschen Krebsgesellschaft, Berlin, Deutschland

\begin{abstract}
Das Jahr 2021 bringt 10 Jahre AMNOGProzess und eine neue Bundesregierung: gleich doppelt Grund, zu rekapitulieren, wo wir stehen, was erreicht wurde und wohin die weitere Reise bei der frühen Nutzenbewertung in Deutschland gehen sollte. Aus Sicht der Hersteller von Onkologika hat sich der AMNOG-Prozess als ein stetig hinzulernendes System in wesentlichen Teilen bewährt - was nicht heißt, dass es keinen Weiterentwicklungsbedarf mehr gibt.
\end{abstract}

Die COVID-19-Pandemie hat auch im Jahr 2021 vieles überschattet. Aber ein rundes Jubiläum einer tief greifenden gesundheitspolitischen Neuordnung sollte dennoch nicht einfach unbeachtet vorbeiziehen. Am 1. Januar 2021 jährte sich das Inkrafttreten des Arzneimittelmarktneuordnungsgesetz (AMNOG) zum 10. Mal [1]. Das AMNOG steht insbesondere für die Einführung der "frühen Nutzenbewertung", der deutschen Spielart des Health Technology Assessment (HTA). Deutschland war nicht das erste Land, das HTA eingeführt hat, aber es wurde mit dem AMNOG-Prozess und seinen Instanzen, allen voran der Gemeinsame Bundesausschuss (G-BA) und das Institut für Qualität und Wirtschaftlichkeit im Gesundheitswesen (IQWiG), rasch zu einem viel beachteten Vorreiter weltweit.

Das AMNOG-Verfahren schließt zeitlich an die arzneimittelrechtliche Zulassung bei der Europäischen Arzneimittelagentur (EMA) an. Ziel ist die Bestimmung des Zusatznutzens eines neuen Medikaments im Vergleich zu einem Therapiestandard, der „zweckmäßigen Vergleichstherapie“
(zVT). Der pharmazeutische Unternehmer $(\mathrm{pU})$ reicht dazu mit Markteintritt ein Dossier ein, das vom IQWiG bewertet wird, wofür das Institut 3 Monate Zeit hat. („Markteintritt" ist dabei definiert als Zeitpunkt der erstmaligen Inverkehrbringung eines Medikaments, alternativ als 1 Monat nach Zulassungserweiterung bei Medikamenten, die nach dem 1. Januar 2011 erstmals zugelassen wurden und bei denen bereits ein nutzenbewertetes Anwendungsgebiet existiert.) Auf Basis der IQWiG-Dossierempfehlung und eines Stellungnahmeverfahrens mit mündlicher Anhörung vergibt der G-BA einen Zusatznutzen in 6 Kategorien ( $\mathbb{A b b} 1$ und 2; - Tab. 1).

Die Kategorisierung des Zusatznutzens liefert die Grundlage für die Verhandlung des Erstattungsbetrags zwischen dem Spitzenverband der gesetzlichen Krankenversicherung (GKV-SV) und dem $\mathrm{pU}$, die 6 Monate nach G-BA-Beschluss bzw. wenn die Schiedsstelle angerufen wird, bis 15 Monate nach G-BA-Beschluss, abgeschlossen sein muss. Für 12 Monate ab Markteintritt gilt eine freie Preisbildung. Ab Monat 13 nach erstmaligem Inverkehrbringen bzw. - im Falle einer Indikationserweiterung - Zulassung des neuen Anwendungsgebietes gilt der vereinbarte bzw. geschiedste Erstattungsbetrag. Wichtig ist anzumerken, dass das AMNOG-Verfahren - im Gegensatz zur EMA-Zulassung - nichts über die grundsätzliche Wirksamkeit und Verträglichkeit einer Behandlung aussagt. Es ist kein zweites Zulassungsverfahren und nimmt keine Nutzen-Risiko-Bewertung vor, sondern dient ausschließlich als Grundlage 


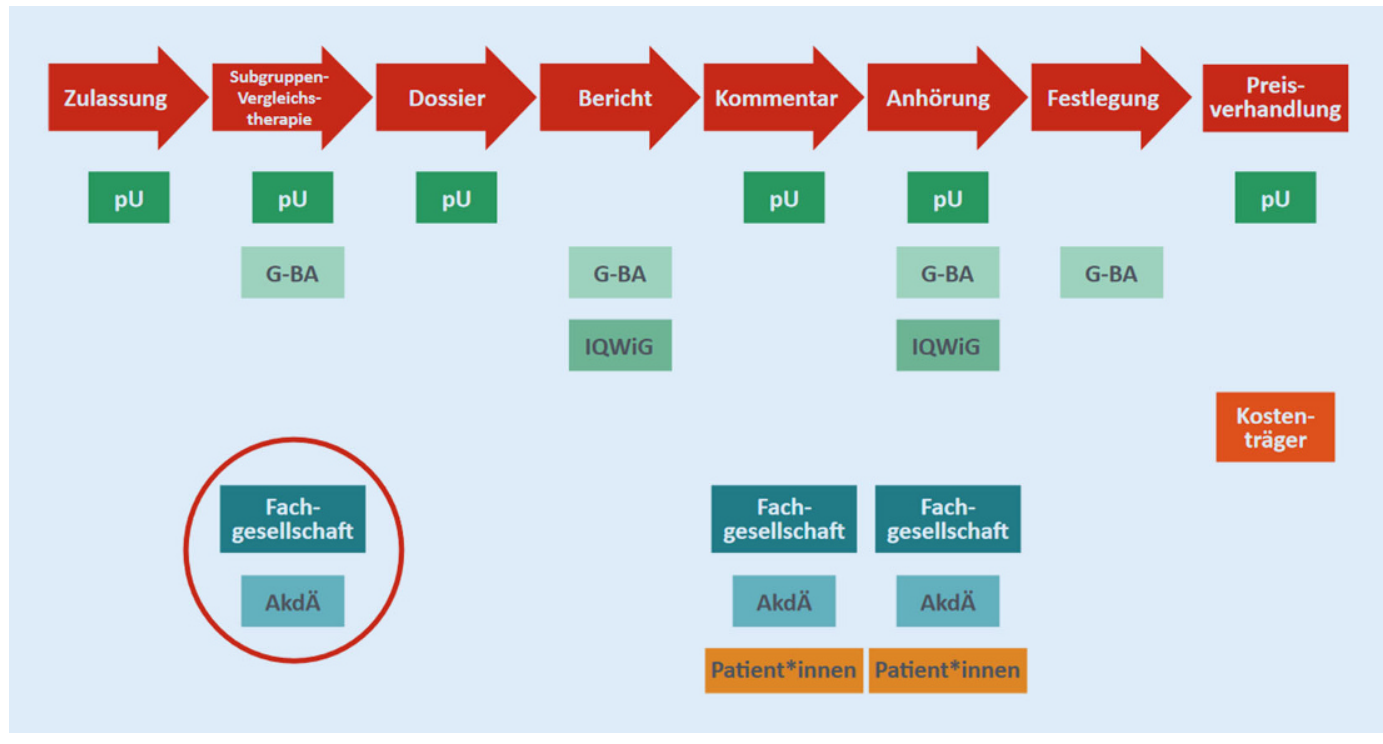

Abb. $1 \triangleleft$ Ablaufschema der frühen Nutzenbewertung nach AMNOG. (Modifiziert nach [2]). AkdÄ Arzneimittelkommission der deutschen Ärzteschaft, G-BA Gemeinsamer Bundesausschuss, IQWiG Institut für Qualität und Wirtschaftlichkeit im Gesundheitswesen, $p U$ pharmazeutisches Unternehmen

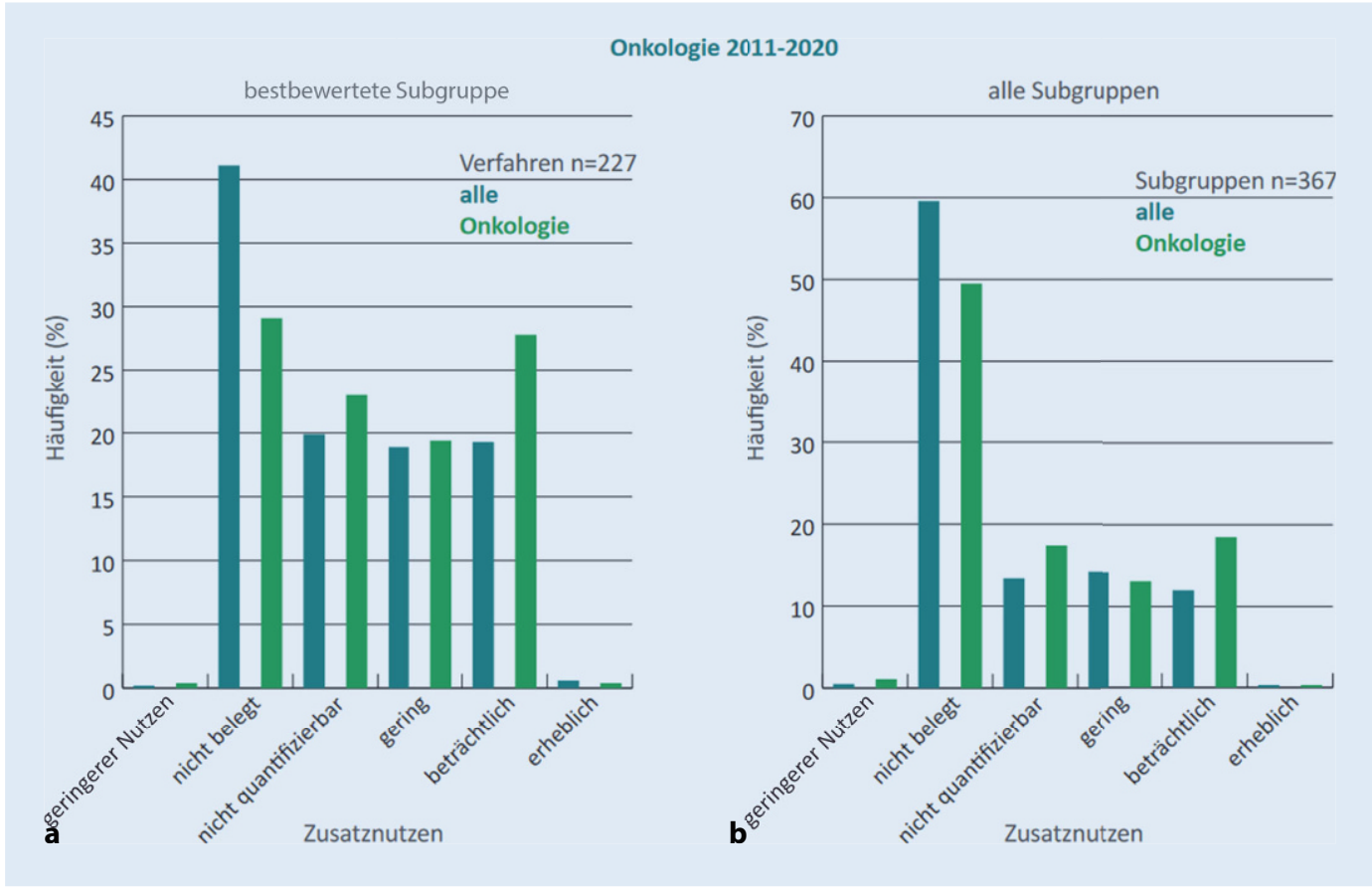

Abb. $2 \triangleleft$ Die Onkologie ist in der deutschen Nutzenbewertung das Fach mit den meisten neuen Arzneimitteln bzw. Indikationserweiterungen. Die Kategorie „beträchtlicher Zusatznutzen" wird in onkologischen Nutzenbewertungen überdurchschnittlich häufig erreicht. (Modifiziert nach [2-4])

für eine zusatznutzenbasierte Preisermittlung. Entsprechend tangiert der G-BABeschluss auch nicht die Zulassung: Das jeweilige Präparat bleibt unabhängig von der Zusatznutzenkategorie uneingeschränkt verordnungsfähig.

\section{Onkologika im AMNOG-Verfahren: Bewährungsprobe bestanden}

Das AMNOG-Verfahren betrifft alle medizinischen Fachrichtungen, aber es hat für jede Fachrichtung andere Herausforderungen zu bewältigen. Ziel dieses Beitrags ist eine Bewertung des ersten AMNOG-Jahrzehnts aus Sicht der Krebsversorgung. In Deutschland war es - gerade mit Blick auf Krebspatienten - von Anfang an politischer Anspruch, dass eine Nutzenbewertung nicht dazu führen darf, dass zugelassene Medikamente erst Monate oder gar Jahre nach Zulassung verfügbar werden.

Das zu verhindern, ist gelungen, was eine sehr gute Nachricht für alle onkologischen Patienten ist. Denn diese haben oft schlicht nicht die Zeit, auf eine verzögerte Markteinführung zu warten. Eine Selbstverständlichkeit ist eine schnelle
Verfügbarkeit von neuen Medikamenten nicht, wie die jährliche WAIT-Befragung des europäischen Pharmaverbands EFPIA gerade erst wieder gezeigt hat. Dort belegte Deutschland im Jahr 2020 mit im Median 50 Tagen zwischen Zulassung und Verordnungsfähigkeit in Europa Platz 1. In der Schweiz waren es 87, in Italien 357 und in Frankreich 474 Tage [5].

Beispiele der letzten Jahre mit onkologischem Bezug, die diese klare Stärke des deutschen AMNOG-Prozesses illustrieren und um die die Bundesrepublik im Übrigen auch international beneidet wird - gibt es 
Tab. 1 Nutzenbewertungsverfahren in Deutschland 2011-2020. Der G-BA vergibt den Zusatznutzen (ZN) in 6 Kategorien [4]

\begin{tabular}{|l|l|}
\hline Zusatznutzenkategorie & Definition \\
\hline Erheblicher ZN & $\begin{array}{l}\text { Nachhaltige und bisher nicht erreichte große Verbesserung des } \\
\text { therapierelevanten Nutzens }\end{array}$ \\
\hline Beträchtlicher ZN & $\begin{array}{l}\text { Bisher nicht erreichte deutliche Verbesserung des therapierelevan- } \\
\text { ten Nutzens }\end{array}$ \\
\hline Geringer ZN & $\begin{array}{l}\text { Bisher nicht erreichte moderate und nicht nur geringfügige Ver- } \\
\text { besserung des therapierelevanten Nutzens }\end{array}$ \\
\hline Nicht quantifizierbarer ZN & Wissenschaftliche Datengrundlage lässt keine Quantifizierung zu \\
\hline ZN nicht belegt & Kein Zusatznutzen belegt \\
\hline Geringerer Nutzen & Nutzen ist geringer als der der ZVT \\
\hline zVT zweckmäßige Vergleichstherapie \\
\hline
\end{tabular}

reichlich. Genannt sei die konsequent umgesetzte Einführung von Immunonkologika wie 2011 Ipilimumab sowie 2015 Nivolumab und Pembrolizumab - eine komplett neue Säule der Krebstherapie, die das System vorher nicht kannte. Zu nennen wären auch die Tyrosinkinaseinhibitoren sowie die „advanced therapy medicinal products" (ATMP), wobei bei Letzteren die "Einführungsphase" ins AMNOG-System noch läuft und sicher noch die eine oder andere Stellschraube zu betätigen ist. Dennoch: Dass auch erste onkologische CAR-T-Zelltherapien, wie Tisagenlecleucel und Axicabtagene Ciloleucel, zügig Einzug in die reguläre Versorgung und die GKVErstattung gehalten haben und dass damit gelungen ist, Maßnahmen zur Qualitätssicherung zu formulieren [6], all das spricht für die Leistungs- und Adaptionsfähigkeit des AMNOG-Prozesses.

\section{Das AMNOG als lernendes System}

Die Adaptationsfähigkeit des AMNOG-Prozesses scheint uns rückblickend ohnehin der vielleicht entscheidende Faktor dafür gewesen zu sein, dass das deutsche HTA vergleichsweise erfolgreiche 10 Jahre hinter sich hat. Onkologische Fachgesellschaften in der Arbeitsgemeinschaft der Wissenschaftlichen Medizinischen Fachgesellschaften (AWMF) haben, genauso wie Vertreter aus Politik, Medizin, Wissenschaft und Wirtschaft, schon früh darauf gedrungen, den AMNOG-Prozess als ein lernendes System zu begreifen, das über die Jahre weiterentwickelt werden muss.

Dies war anfangs durchaus nicht Konsens, aber hat sich als richtig und wichtig herausgestellt. Ablesbar ist der kon- tinuierliche Lernprozess nicht zuletzt an der langen Liste von Gesetzen mit Bezug zur frühen Nutzenbewertung, die auf das AMNOG folgten. Genannt seien hier beispielhaft das Gesetz zur Stärkung der Arzneimittelversorgung in der GKV (GKVAMVSG) aus dem Jahr 2017, das die Bündelung mehrerer Bewertungsverfahren zu einem Wirkstoff und die Abbildung von Nutzenbewertungsbeschlüssen in der Praxissoftware brachte oder das Gesetz für mehr Sicherheit in der Arzneimittelversorgung (GSAV) aus dem Jahr 2019, das die anwendungsbegleitende Datenerhebung und eine stärkere Beteiligung der Fachgesellschaften einführte. Außerhalb der Onkologie hat das Gesetz für einen fairen Kassenwettbewerb in der gesetzlichen Krankenversicherung (GKV-FKG) aus dem Jahr 2020 klargestellt, dass für freigestellte Reserveantibiotika ein Zusatznutzen als belegt gilt.

Die Vielzahl an G-BA-Entscheidungen eines Jahrzehnts zu würdigen, sprengt auch dann den Rahmen dieses Beitrags, wenn wir uns auf die Onkologie fokussieren. Ein Blick in den Geschäftsbericht 2020 des G-BA genügt, um zu illustrieren, wie aussichtslos ein solches Unterfangen wäre. Allein in diesem einen (Corona-)Jahr haben die pU beim G-BA 116 Nutzendossiers eingereicht, mehr als je zuvor. Allein 42 davon kamen aus dem Bereich Onkologie [7]. Auch in den 9 Jahren davor war die Onkologie bei den Nutzenbewertungen mit Abstand Spitzenreiter (ब Tab. 2). Vor diesem Hintergrund sollen im Rahmen dieses Beitrags weniger einzelne Entscheidungen als einige der "strukturellen Neuerungen" hervorgehoben werden, die den AMNOG-Prozess in den letzten $10 \mathrm{Jah}$ -
Tab. 2 Anzahl vollständig durchgeführter, früher Nutzenbewertungen nach Fachgebieten. (Modifiziert nach [2])

\begin{tabular}{|l|l|l|}
\hline Fachgebiet & $\begin{array}{l}\mathbf{2 0 1 1 -} \\
\mathbf{2 0 1 8}\end{array}$ & $\begin{array}{l}\mathbf{2 0 1 9 /} \\
\mathbf{2 0 2 0}\end{array}$ \\
\hline Dermatologie & 35 & 15 \\
\hline Diabetologie & 36 & 9 \\
\hline Endokrinologie & 46 & 10 \\
\hline Gynäkologie & 12 & 17 \\
\hline Hämatologie & 59 & 38 \\
\hline Hämostaseologie & 15 & 7 \\
\hline $\begin{array}{l}\text { Hals-Nasen-Ohren- } \\
\text { Heilkunde }\end{array}$ & 2 & 5 \\
\hline $\begin{array}{l}\text { Hepatogastro- } \\
\text { enterologie }\end{array}$ & 34 & 10 \\
\hline Infektiologie & 36 & 9 \\
\hline Kardiologie & 12 & 4 \\
\hline Nephrologie & 13 & 5 \\
\hline Neurologie & 30 & 14 \\
\hline Onkologie & 139 & 89 \\
\hline Ophthalmologie & 10 & 3 \\
\hline Pädiatrie & 30 & 38 \\
\hline Pneumologie & 47 & 33 \\
\hline Psychiatrie & 5 & 1 \\
\hline Rheumatologie & 6 & 4 \\
\hline Stoffwechsel & 27 & 26 \\
\hline Urologie & 18 & 12 \\
\hline
\end{tabular}

ren als lernendes System ausgezeichnet haben.

\section{Konstruktives Miteinander im Vorfeld der Nutzenbewertung}

Positiv entwickelt haben sich über die Jahre die Möglichkeiten zur gezielten Abstimmung zwischen den $\mathrm{pU}$ und den HTAInstanzen nach § 35a Abs. 7 SGB V. Das betrifft einerseits die "späte“ Beratung zum Zeitpunkt der Dossiererstellung. Insbesondere ist aber hier die "frühe“ Beratung zu Studien sowie zur zVT hervorzuheben. Die mit dem 2. AMGÄndG [8] 2012 eingeführte sukzessive ausgebaute Beteiligung der Zulassungsbehörden an diesen Beratungen hilft, Studien und Studienendpunkte so zu planen, dass den Bedürfnissen sowohl der EMA und des Bundesinstituts für Arzneimittel und Medizinprodukte (BfArM) bzw. Paul-Ehrlich-Instituts (PEI) als auch des G-BA Rechnung getragen wird. Und mit der im Jahr 2019 mit dem GSAV [9] hinzugekommenen Möglichkeit der Beteiligung von Fachgesellschaften bzw. der Arzneimittelkommission der deutschen Ärz- 
teschaft (AkdÄ) öffnet sich der AMNOGProzess weiter, was im Idealfall ein gemeinsames Verständnis für beispielsweise eine geeignete zVT im jeweiligen Anwendungsgebiet fördert.

Dieses gemeinsame Verständnis im Hinblick auf die zVT sollte dann allerdings nicht im Nachhinein wieder infrage gestellt werden. In Situationen, in denen der G-BA mehrere Therapien alternativ als zVT festgelegt hat, sind diese im Rahmen der Nutzenbewertung auch in gleicher Weise als geeignete Komparatoren zum Nachweis eines Zusatznutzens zu behandeln. Es ist nicht hinnehmbar, wenn später, im Rahmen der eigentlichen Nutzenbewertung, plötzlich Kritik an der durch den pU vorgenommenen Auswahl der zVT geübt oder die vom G-BA bei der Studienplanung kommunizierte Auswahlmöglichkeit im Verfahren oder gar nachträglich eingeschränkt wird (siehe etwa G-BA-Anhörung vom 20.08.2020 zu Apalutamid [10]). Hier muss es mit Blick auf die Planungssicherheit für die $\mathrm{pU}$ - und hier v.a. mit Blick auf die bei jeder Arzneimittelentwicklung nötige sehr komplexe internationale Abstimmung von Studiendesigns - die Konsistenz bei der Beibehaltung einer zVT sowie der Flexibilität ergänzender zVTFestlegung über den gesamten AMNOGProzess hinweg geben.

\section{Erstattung der Biomarker- diagnostik ist kein $\mathrm{K}(\mathrm{r}) \mathrm{ampf}$ mehr}

Sehr positiv zu bewerten ist aus Sicht insbesondere der onkologischen Versorgung, dass es durch § 87 Abs. 5b SGB V im Rahmen des GKV-AMVSG im Jahr 2017 gelungen ist, für die immer wichtiger werdende, i. d. R. ambulante Biomarkerdiagnostik einen effizienten Modus der Abrechenbarkeit zu etablieren. Der Trend zur wachsenden Biomarkerdiagnostik ist der zunehmenden Verfügbarkeit zielgerichteter Antitumortherapien zu verdanken. Durch die Biomarkertests werden u.a. jene Krebspatienten identifiziert, die von einer gezielten Antitumortherapie mit hoher Wahrscheinlichkeit profitieren. Ein Präzedenzfall war die EGFR-Testung bei den EGFR-Inhibitoren, die anfangs teilweise von den $\mathrm{pU}$ bezahlt wurde, da sie im Erstattungssys- tem vor AMNOG-Zeiten nicht vorgesehen war.

Das hat sich geändert: Konkret wird seit 2017 bei all jenen onkologischen Präparaten, bei denen in der Zulassung ein diagnostischer Marker explizit genannt wird, automatisch mit Dossiereinreichung ein Prozess angestoßen, der zu einer Anpassung des für die ambulante Abrechenbarkeit maßgeblichen einheitlichen Bewertungsmaßstabs (EBM) zeitgleich mit der Finalisierung des Nutzenbewertungsbeschlusses führt. Dieser pragmatische Weg in die Biomarkererstattung ist zielführend. Und er ist auch wirtschaftlich und medizinisch sinnvoll, weil er für die Erstattung der Testung quasi das Primat der Therapie etabliert: Es werden nicht Tests in den Markt gedrückt, die eine Therapie „suchen", sondern es werden erstattungsfähige Tests dann zur Verfügung gestellt, wenn sie tatsächlich sinnvoll bzw. qua Zulassung erforderlich sind. Demgegenüber gibt es im stationären Sektor allerdings weiterhin keine regelhafte Erstattung der Biomarkerdiagnostik.

\section{Endpunkte in der Evidenz- bewertung}

Was die vom G-BA anerkannte Evidenz angeht, gibt es in der Onkologie die das gesamte erste AMNOG-Jahrzehnt begleitende Diskussion um das progressionsfreie Überleben (PFS), dem das IQWiG weiterhin nicht die Bedeutung als patientenrelevanter Endpunkt zuschreibt und über dessen Patientenrelevanz innerhalb des G-BA Uneinigkeit besteht. In diesem Punkt gibt es einen Dissens zwischen der Mehrheit der pU, den Fachgesellschaften und vielen Patientenvertretern einerseits sowie den bundesdeutschen HTA-Instanzen andererseits. Insgesamt erscheint es uns wichtig zu betonen, dass eine Nutzenbewertung nicht übersimplifizieren darf. Vielmehr sollten patientenrelevante Endpunkte tumorentitäts- und stadienabhängig ausgewählt werden, um der Komplexität der Krebstherapie Rechnung zu tragen [3].

Ein in diese Richtung gehender Lernprozess des AMNOG bei der Evidenzbewertung ist - bei allem Dissens in puncto PFS - in der Gesamtschau klar zu erkennen. Zu nennen ist hier die Anerkennung bildgebender intermediärer Endpunkte in bestimmten Therapiekonstellationen. Besonders wichtig ist das in der adjuvanten Krebstherapie, wo der G-BA bei tumorfreien Patienten das Tumorrezidiv auch dann als patientenrelevant anerkennt, wenn es ausschließlich über Biomarker bzw. Bildgebung diagnostiziert wird und noch nicht klinisch manifest ist. So wurden in der jüngeren Vergangenheit sowohl bei Tyrosinkinaseinhibitoren (BRAF/MEK) als auch bei Immuntherapeutika (PD-1) radiologisch manifest gewordene Rezidive anerkannt [11, 12]. Aber auch in hämatologisch-onkologischen Indikationen wurde mit dem ereignisfreien Überleben ein solcher Endpunkt vom G-BA anerkannt, erstmalig konkret im Nutzenbewertungsverfahren zu Brentuximab-Vedotin beim anaplastischen, großzelligen Lymphom [13].

\section{Prozessinnovationen in der Evidenzbewertung}

Daneben sind im Zusammenhang mit Evidenz und Evidenzbewertung einige nur scheinbar nebensächliche Prozessinnovationen hervorzuheben, die den AMNOGAlltag aus Sicht der pU zum Positiven verändert haben. So brachte das GKV-AMVSG im Jahr 2017 die Möglichkeit, eine erneute Nutzenbewertung aufgrund neuer wissenschaftlicher Erkenntnisse bereits vor Ablauf eines Jahres beim G-BA zu beantragen. Das führt zu einer gewissen Beschleunigung in Konstellationen mit sich dynamisch verändernder Evidenzsituation, wobei das Wiederbewertungsverfahren dann trotzdem erst nach Ablauf des Jahres startet.

Hilfreich ist die ebenfalls mit dem GKVAMVSG geschaffene Möglichkeit der Bündelung von mehreren Nutzenbewertungsverfahren zu einem Arzneimittel mit neuem Wirkstoff. Die Bündelungsoption ermöglicht es dem pU, Dossiers bei der Einreichung „zusammenzulegen“, wenn innerhalb von 6 Monaten nach Markteintritt die Zulassung weiterer Anwendungsgebiete zu erwarten ist. Auf diese Weise kann der pU im Einzelfall von gesetzlichen Fristvorgaben abweichen und erforderliche Nachweise zur Nutzenbewertung "gebündelt" einreichen. Auch das führt letztlich zu einer Steigerung der Verfah- 
renseffizienz aufseiten von G-BA, GKV-SV und pU.

Positiv hervorgehoben werden sollte an dieser Stelle auch, dass der G-BA bisher allerdings nur in Ausnahmefällen - indirekte Vergleiche im Kontext einer Nutzenbewertung akzeptiert. Bei einem solchen indirekten Vergleich wird der Zusatznutzen im Vergleich zu einer zVT quasi über Studiengrenzen hinweg beurteilt. Indirekte Vergleiche sind immer dann sinnvoll, wenn randomisierte klinische Studien nicht möglich oder auch ethisch nicht geboten sind. Insbesondere bei selten auftretenden Erkrankungen mit kleinen Patientenzahlen, einer hohen Krankheitslast und mangelnden therapeutischen Behandlungsoptionen ist die Evidenzgenerierung über indirekte Vergleiche sinnvoll. Dies war etwa bei der Nutzenbewertung von Pembrolizumab in Kombination mit Pemetrexed und Platin beim nichtkleinzelligen Lungenkarzinom derFall; hier konnte die Überlegenheitüber einen indirekten Vergleich im Vergleich zur Monotherapie über den „Brückenkomparator" Chemotherapie gezeigt werden [14].

\section{Nutzen aus Patientensicht findet Berücksichtigung}

Eine deutliche Weiterentwicklung hat der AMNOG-Prozess gerade in den letzten Jahren im Bereich patientenberichteter Endpunkte (PRO) erlebt. Die PRO werden über standardisierte und validierte Fragebögen von den Patienten selbst erhoben bzw. dokumentiert. Mittels patientenberichteter Symptome kann ein Zusatznutzen in der Kategorie Morbidität und über patientenberichtete psychosoziale und Verhaltensaspekte in der Kategorie gesundheitsbezogene Lebensqualität erreicht werden. Zwar waren patientenberichtete Symptome (als Endpunkte zur Morbidität) und gesundheitsbezogene Lebensqualität - neben Mortalität und Sicherheit - von Anfang an als Nutzenkategorien im Sinne des AMNOG-Prozesses vorgesehen. Ihre Bedeutung trat allerdings in den ersten Jahren deutlich hinter eine demgegenüber sehr stark betonte Mortalitätssenkung zurück, was v.a. in der metastasierten Therapiesituation wiederholt Schwierigkeiten bereitet hat.
Einen wichtigen Durchbruch in Richtung einer besseren Würdigung von PRO bei der Nutzenbewertung bildete das initiale Nutzenbewertungsverfahren für Crizotinib beim ALK-positiven fortgeschrittenen nichtkleinzelligen Lungenkarzinom [15]. Als Beispiel für die Einbeziehung von PRO in die Nutzenbewertung bei hämatologischen Neoplasien kann das AMNOGVerfahren zu Carfilzomib in Kombination mit Dexamethason bzw. Lenalidomid/ Dexamethason beim vortherapierten multiplen Myelom genannt werden [16]. Hier kam als PRO für die Erfassung von Symptomen und gesundheitsbezogener Lebensqualität der mit onkologischen Patienten entwickelte etablierte Lebensqualitätsfragebogen EORTC QLQ-C30 zum Einsatz, außerdem der myelomspezifische EORTCQLQ-MY20. Insgesamt ist die Erhebung von PRO bei vielen Antitumortherapien essenziell für das Verständnis der Patientenperspektive, umso mehr zu begrüßen ist die zunehmende Berücksichtigung in den Nutzenbewertungen.

\section{Offene Punkte in der neuen Legislatur}

Anzuerkennen, dass das AMNOG-Verfahren ein lernendes System ist, beinhaltet auch, dass der Lernprozess ein kontinuierlicher ist und nicht im 2. Lebensjahrzehnt oder mit Ablauf einer Legislatur endet. An dieser Stelle sollen deswegen einige aktuell offene Punkte genannt werden, die aus Sicht der in der Onkologie tätigen pU an eine neue Bundesregierung adressiert werden.

- Evidenztransfer: Die Übertragung von Evidenz von bestimmten Patientengruppen auf andere ist im AMNOG-Prozess vorgesehen und gesetzlich geregelt. Bei Arzneimitteln mit einer Genehmigung für die pädiatrische Verwendung (,PUMAArzneimittel ${ }^{\prime}$ ) arbeitet der G-BA derzeit an einer Verfahrensordnung, die bei der Nutzenbewertung einen besseren Evidenztransfer in Richtung solcher Patientengruppen möglich machen soll, die von der Zulassung zwar abgedeckt, in den jeweiligen Studien aber nicht in ausreichenden Umfang erfasst werden konnten. Eine derartige Übertragung von Evidenz kann auch in der Erwach- senenonkologie und bei Paediatric investigation plan(PIP)-basierten $\mathrm{Zu}$ lassungserweiterungen zielführend sein. Wichtig ist dabei ein klar definiertes, methodisches Regelwerk zur Umsetzung eines solchen Transfers, um die nötige breite Akzeptanz zu erreichen.

- Responderschwellen bei der PROErfassung: Dies ist ein wichtiges Thema und Gegenstand vieler aktueller Diskussionen zwischen den HTA-Instanzen, den Fachgesellschaften und den pU. Konkret geht es um die Frage, wie viel prozentuale Veränderung bei PRO für die Nutzenbewertung als relevant anzusehen ist. Das IQWiG plädiert in seinem Methodenpapier [17], basierend auf mehreren Übersichtsarbeiten aus unterschiedlichen Indikationsbereichen, für einen allgemeinen und indikationsgebietsunabhängigen Schwellenwert von $15 \%$ der jeweiligen Skalenspannweite. Kritiker monieren, dass ein solches pauschales Vorgehen der PRO-Erfassung nicht gerecht wird und schlicht eine erfahrungsbasierte Festlegung darstellt. Im Rahmen der Nutzenbewertung sollten wissenschaftlich etablierte Schwellen nach Ansicht der pU weiterhin akzeptiert werden. Die wissenschaftliche Evaluation weiterer Schwellen sollte dabei weiterhin unabhängig vom AMNOG erfolgen.

- Dokumentationsbürokratie: Hinsichtlich der für ein HTA-Verfahren erforderlichen Bürokratie besteht Handlungsbedarf. Das betrifft in erster Linie die neuen Dossiervorlagen, die der G-BA per Beschluss vom 20. Juni 2019 eingeführt hat und die seit April 2020 verpflichtend genutzt werden müssen. Deren Analyseumfang hat im Vergleich zu den bisherigen Dossiervorlagen stark zugenommen, exemplarisch festzumachen an den Anforderungen für nicht prädefinierte Subgruppenanalysen ohne signifikante Interaktion, die den Aufwand für die pU deutlich vergrößert haben - ohne aber bislang für die Nutzenbewertung relevant gewesen zu sein.

- Einbeziehung versorgungsnaher Daten: Die Berücksichtigung versorgungsnaher Daten in der Evidenzbewertung 
ist eine noch junge "AMNOG-Neuerung", die mit dem GSAV im Jahr 2019 in die Nutzenbewertung kam. Die pharmazeutische Industrie begrüßt diese Neuerung grundsätzlich, stellt aber klar, dass diese eindeutig definierten Ausnahmekonstellationen vorbehalten bleiben soll. Exemplarisch niedergelegt wurde ein zielführendes Verfahren für die Etablierung Wissen generierender Register in einem Methodenleitfaden des Deutschen Netzwerks Versorgungsforschung [18]. Anders als die vom G-BA mit Beschluss vom 16. Juli 2020 definierte Verfahrensordnung [19] enthält es einen transparenten Konsensprozess. Unstrittig zwischen allen Beteiligten ist hingegen, dass Register für eine Nutzenbewertung prospektiv sein und auch sonst hohen Qualitätsstandards genügen müssen. Sie sollten unabhängig und außerhalb des AMNOG-Prozesses aufgesetzt werden. Die klinischen Krebsregister kämen dafür prinzipiell infrage, sind aber aktuell für die Zwecke der Nutzenbewertung noch nicht umfassend genug, sodass bis auf Weiteres ergänzende prospektive Register nötig sind.

- Weiterentwicklung der Evidenzanforderungen: In den vergangenen 10 Jahren wurden die initial eher starren Evidenzanforderungen im AMNOGProzess an einigen Stellen flexibilisiert. Um diesen Weg weiterzugehen, kann ein Diskussionspunkt hierbei die Erweiterung der vom G-BA akzeptierten patientenrelevanten Endpunkte um klinisch bereits etablierte morbiditätsbezogene Endpunkte sein. So ist beispielsweise das metastasenfreie Überleben ein von Zulassungsbehörden anerkannter Endpunkt beim fortgeschrittenen Prostatakarzinom. Und Endpunkte wie die Zeit bis zur nächsten Folgetherapie („time to subsequent therapy ${ }^{\prime \prime}$ ) oder die Zeit bis zur ersten Chemotherapie gewinnen nach Ansicht der pU ihre Patientenrelevanz dadurch, dass sie ein Maß dafür sind, wie weit belastende Folgeinterventionen hinausgezögert werden können. An dieser Stelle sei angemerkt, dass auch für die mittlerweile akzeptierten Endpunkte des ereignis-/rezidivfreien
Überlebens in der Adjuvans noch insofern Klärungsbedarf besteht, als teilweise infrage gestellt wird, welche Art von Behandlung als kurativ anzusehen ist und damit die Nutzung dieses Endpunkts ermöglicht.

- Nutzenbewertung bei tumorübergreifenden Zulassungen: Die klinische Forschung ist bei Tumoren, die durch seltene Mutationen ausgelöst werden, in einem ethischen Dilemma. Hier gibt es oft keine spezifischen medikamentösen Behandlungsoptionen, gleichzeitig sind große PhaseIII-Studien praktisch nicht möglich. Allerdings besteht die Möglichkeit, in solchen Fällen Evidenz über einarmige, tumorübergreifende Phase-II-Studien zur Sicherheit und Wirksamkeit zu generieren. Liegen aus solchen „BasketStudien" belastbare Ergebnisse vor, hat die EMA bereits in der Vergangenheit Zulassungen erteilt. Bei den bisherigen Nutzenbewertungen zu onkologischen Substanzen mit tumorübergreifender Zulassung, Entrectinib [20] und Larotrectinib [21], hat sich der durch die Zulassung erwiesene Nutzen dieser Substanzen jedoch nicht in einen Zusatznutzen übersetzt. Einen Weg zur Berücksichtigung der Besonderheiten tumorübergreifender Zulassungen im Rahmen der Nutzenbewertung stellt - neben der Darstellung tumorübergreifender Analysen der Gesamtpopulation die Betrachtung der Datenlage für einzelne Tumorentitäten, sog. Leitentitäten, dar. Die indirekt vergleichende Bewertung erfolgt dann auf Basis von Daten, die für Leitentitäten erhoben wurden. Allerdings gilt auch für dieses Vorgehen die Herausforderung der kleinen Populationen, da Leitentitäten Subpopulationen der oftmals bereits sehr kleinen, tumoragnostischen Zielpopulation darstellen.

- Nutzenbewertung von Arzneimitteln für neuartige Therapien (ATMP): ATMP sind Arzneimittel für die Anwendung beim Menschen, die auf Genen, Geweben oder Zellen basieren. Grundsätzlich ist der AMNOGProzess auch für ATMP ein geeigneter Regulierungsrahmen, den es allerdings in den kommenden Jahren noch weiter auszugestalten gilt. Oben geschilderte Herausforderungen im Zusammenhang mit unterschiedlichen Evidenzanforderungen von EMA und G-BA gelten hier in besonderem Maße. Registern und indirekten Vergleichen kommt bei den im AMTP-Kontext oft seltenen Erkrankungen eine hohe Bedeutung zu. Jenseits von Fragen der Evidenzbewertung ist außerdem grundsätzlich bislang nicht geklärt, wie im Rahmen des AMNOG-Prozesses und der anschließenden Preisverhandlungen mit Einmaltherapien umzugehen ist. Für die Planungssicherheit wäre eine Ausgestaltung des entsprechenden Regelwerks wünschenswert.

- Governance: Abschließend sei darauf hingewiesen, dass es im AMNOGProzess seit seiner Implementierung eine bis heute aus Sicht der pU nicht zufriedenstellend gelöste GovernanceProblematik gibt, die aus einer „Doppelrolle" des GKV-SV herrührt. Der GKVSV ist ein maßgeblicher Akteur bei der Festlegung des Zusatznutzens durch den G-BA, gleichzeitig ist er der Verhandlungspartner der $\mathrm{pU}$ bei den sich anschließenden Preisverhandlungen.

\section{Fazit}

Insgesamt lässt sich konstatieren, dass sich im AMNOG-Prozess ein konstruktives, Stakeholder-übergreifendes Kommunikationsklima entwickelt hat, das dem Erfordernis einer gewissen Planungssicherheit seitens der pU versucht, Rechnung zu tragen, und auch vertrauensbildend gewirkt hat. Die Einführung eines HTA-Systems in Deutschland ist aus dem Blickwinkel der Krebsversorgung im Wesentlichen nicht mit einer Verschlechterung des Zugangs zu Onkologika einhergegangen. Dies sollten wir unbedingt zu erhalten versuchen, auch wenn der in der Krebsmedizin bevorstehende Innovationsschub und die Bewältigung der finanziellen Pandemiefolgen parallele Herausforderungen darstellen.

Die bisherigen Strukturen des AMNOGProzesses sind in weiten Teilen entwickelt worden, als noch niemand Immunonkologika mit ihrer Vielzahl von Indikationserweiterungen auf der einen sowie Genund Zelltherapien auf der anderen Seite 
kommen sah, als es noch keine tumorübergreifenden Arzneimittelzulassungen gab und als sich noch kaum jemand vorstellen konnte, in welchem Umfang etablierte Tumorentitäten durch molekulargenetische Marker innerhalb weniger Jahre in teils Dutzende Subentitäten aufgespaltet würden.

Es wäre allerdings nicht fair, so zu tun, als ob einfach nur die HTA-Instanzen ihre Hausaufgaben machen müssten. Tatsächlich entwickeln sich Medizin und Wissenschaft insgesamt weiter. Die eigentliche Botschaft, die von diesem Beitrag ausgehen sollte, ist daher eine breitere: Ein Gesundheitswesen, das den Anspruch erhebt, eines der besten weltweit zu sein, und dies auch bleiben möchte, muss den medizinisch-wissenschaftlichen Fortschritt insgesamt im Blick behalten und, wenn nötig, seine Strukturen und seine Bewertungssystematiken kontinuierlich anpassen.

\section{Korrespondenzadresse}

\section{Dr. Cornelia Lechner}

Arbeitsgruppe Gesundheitspolitik und Market Access der Sektion C der Deutschen Krebsgesellschaft

Berlin, Deutschland

clechner@amgen.com

Danksagung. Die Autoren danken Herrn Philipp Grätzel von Grätz, Berlin, für die Entwicklung und das Redigieren des Manuskripts, das seitens der Sektion C der Deutschen Krebsgesellschaft finanziell unterstützt wurde.

\section{Einhaltung ethischer Richtlinien}

Interessenkonflikt. A. Bartol ist als Angestellter beschäftigt bei der Janssen-Cilag GmbH, Neuss, Deutschland. K. Dreßler ist als Angestellte beschäftigt bei der Bayer Vital GmbH, Berlin, Deutschland. P. Kaskel warzur Zeit der Konzeptentwicklung und dessen Umsetzung als Angestellter der Firma MSD SHARP \& DOHME GMBH beschäftigt, einer deutschen Tochtergesellschaft von MERCK \& CO., INC., Kenilworth, NJ, USA. C. Landsberg ist als Angestellte beschäftigt bei der Novartis Pharma $\mathrm{GmbH}$. C. Lechner ist als Angestellte beschäftigt bei der Amgen GmbH. M. Petschulies ist in Vollzeit bei der Takeda Pharma Vertrieb GmbH \& Co. KG angestellt.

Für diesen Beitrag wurden von den Autoren keine Studien an Menschen oder Tieren durchgeführt. Für die aufgeführten Studien gelten die jeweils dort angegebenen ethischen Richtlinien.

\section{Literatur}

1. Gesetz zur Neuordnung des Arzneimittelmarktes in der gesetzlichen Krankenversicherung (ArzneimittelmarktneuordnungsgesetzAMNOG). Bundesgesetzblatt Teil I; 2010; Nr. 67 vom 27.12.2010. https://www.bgbl.de/xaver/ bgbl/start.xav?start=\%2F\%2F*[\%40attr_id\%3D \%27bgbl110s2262.pdf\%27]\#_bgbl_\%2F\%2F* \%5B\%40attr_id\%3D\%27bgbl110s2262.pdf\%27 \%5D_1631798366078. Zugegriffen: 16. Sept. 2021

2. Deutsche Gesellschaft für Hämatologie und Onkologie (DGHO) (2021) https://www.dgho. de/publikationen/schriftenreihen/fruehenutzenbewertung/awmf_amnog_2021 210x297_ok_ansicht_es.pdf. Zugegriffen: 16. Aug. 2021

3. Dabisch letal (2014) Health Econ Rev 4:2

4. Gemeinsamer Bundesausschuss (G-BA) Zusatznutzen neuer Arzneimittel. https://www.g-ba. de/themen/arzneimittel/arzneimittel-richtlinieanlagen/nutzenbewertung-35a/zusatznutzen. Zugegriffen: 16. Aug. 2021

5. Verband der forschenden Pharma-Unternehmen (vfa) (2021) Deutschland ist schnell. Pressemeldung 25. Mai

6. https://www.g-ba.de/beschluesse/4477/. Zugegriffen: 16. Sept. 2021

7. Gemeinsamer Bundesausschuss Geschäftsbericht 2020. https://www.g-ba.de/downloads/1798-5148/2021-07-01_G-BA_Geschaeftsbericht 2020_bf.pdf.Zugegriffen: 10.Aug. 2021

8. Zweites Gesetz zur Änderung arzneimittelrechtlicher und anderer Vorschriften ( 2. AMGÄndG). Bundesgesetzblatt Teil l; 2012; Nr. 50 vom 25.10.2012. https://www.bgbl.de/xaver/ bgbl/start.xav?startbk=Bundesanzeiger_BGBI\& start=//*\%5B@attr_id=\%2527bgbl112s2192. pdf\%2527\%5D\#_bgbl_ $\% 2 F \% 2 F * \% 5 B$ \%40attr_id\%3D\%27bgbl112s2192.pdf\%27 $\% 5 D \_1631798595552$. Zugegriffen: 16. Sept. 2021

9. Gesetz für mehr Sicherheit in der Arzneimittelversorgung (GSAV). Bundesgesetzblatt Teil l; 2019; Nr. 30 vom 15.08.2019. https://www.bgbl.de/ xaver/bgbl/start.xav?startbk=Bundesanzeiger_ BGBI\&start=//*[@attr_id=\%27bgbl119s1202. pdf\%27]\#_bgbl_\%2F\%2F*\%5B\%40attr_ id\%3D\%27bgbl119s1202.pdf\%27\%5D _ 1631798626985.Zugegriffen: 16. Sept. 2021

10. Gemeinsamer Bundesausschuss (G-BA) Nutzenbewertungsbeschluss vom 20. August 2020; TOP 8.1.11, ab Minute 47:20. https://www.g-ba.de/ service/livestream-mediathek/. Zugegriffen: 16. Sept. 2021

11. https://www.g-ba.de/bewertungsverfahren/ nutzenbewertung/451/. Zugegriffen: 10. Aug. 2021

12. https://www.g-ba.de/bewertungsverfahren/ nutzenbewertung/387/. Zugegriffen: 10. Aug. 2021

13. https://www.g-ba.de/bewertungsverfahren/ nutzenbewertung/557/. Zugegriffen: 16 . Sept. 2021

14. https://www.g-ba.de/downloads/40-268-6021/ 2019-09-19_AM-RL-XII_Pembrolizumab_D447_TrG.pdf.Zugegriffen: 10.Aug. 2021

15. https://www.g-ba.de/downloads/40-268-2301/ 2013-05-02_AM-RL-XII_Crizotinib_TrG.pdf. Zugegriffen: 10.Aug. 2021

16. https://www.g-ba.de/bewertungsverfahren/ nutzenbewertung/308/. Zugegriffen: 10. Aug. 2021
17. Institut für Qualität und Wirtschaftlichkeit im Gesundheitswesen (IQWiG) (2020) Allgemeine Methoden. Version 6.0 vom 05.11.2020

18. Klinkhammer-Schalke M et al (2020) Manual für Methoden und Nutzung versorgungsnaher Daten zur Wissensgenerierung. Gesundheitswesen 82:716-722

19. Gemeinsamer Bundesausschuss (G-BA) Verfahrensordnung: Verfahren zur Forderung einer anwendungsbegleitenden Datenerhebung. Beschluss vom 16.07.2020. https://www.g-ba.de/ beschluesse/4402/.Zugegriffen: 11. Aug. 2021

20. https://www.g-ba.de/bewertungsverfahren/ nutzenbewertung/588/\#beschluesse-mobile. Zugegriffen: 17. Aug. 2021

21. https://www.g-ba.de/bewertungsverfahren/ nutzenbewertung/502/. Zugegriffen: 17. Aug. 2021 